\title{
Initial periodontal therapy for the treatment of gingival pregnancy tumor
}

\author{
Y.Q. Zhu ${ }^{1,2,3}$, Y.Q. Wang ${ }^{3}$ Y.C. Tang ${ }^{3}$ and C.Z. Li ${ }^{1,2}$
}

${ }^{1}$ The State Key Laboratory Breeding Base of Basic Science of Stomatology (Hubei-MOST) \& Key Laboratory of Oral Biomedicine Ministry of Education, School \& Hospital of Stomatology, Wuhan University, Wuhan, Hubei

Province, China

${ }^{2}$ Department of Periodontology, School \& Hospital of Stomatology, Wuhan University, Wuhan, Hubei Province, China

${ }^{3}$ The Affiliated Huizhou Stomatologic Hospital of Jinan University \& Huizhou Stomatologic Hospital, Huizhou, Guangdong Province, China

Corresponding author: C.Z. Li

E-mail: chengzhangli123@sina.com

Genet. Mol. Res. 15 (2): gmr.15028119

Received December 21, 2015

Accepted February 11, 2016

Published April 27, 2016

DOI http://dx.doi.org/10.4238/gmr.15028119

\begin{abstract}
The purpose of this study was to evaluate the clinical efficacy of initial periodontal therapy in gingival pregnancy tumors. Thirty-nine patients diagnosed with gingival tumors of pregnancy between 2007 and 2015 were enrolled in this study. The patients received initial periodontal therapy, then supportive periodontal therapies at 3to 6-month intervals. The patients underwent follow up for 6 months to 8 years after treatment. After plaque control, supragingival scaling, and root planning, the tumors in 25 patients were gradually eliminated without the necessity of surgery. In 3 patients, tumors $<5 \mathrm{~mm}$ in size disappeared in a mean time of 3.6 months, 4 patients with tumors $5-10 \mathrm{~mm}$ disappeared in a time of 7.5 months, 11 patients with tumors 10-15 mm disappeared in 10.2 months, 6 patients with tumors 15-20 mm disappeared in 15 months, and one patient with a tumor $>20 \mathrm{~mm}$ disappeared in 20 months. No recurrence of gingival pregnancy tumors
\end{abstract}


was noted during subsequent follow-up. Initial periodontal therapy combined with oral hygiene maintenance is efficacious in treating gingival pregnancy tumors of patients with normal hormone levels, which can potentially serve as an option to avoid surgery.

Key words: Gingival pregnancy tumor; Periodontal initial therapy; Oral hygiene; Clinical efficacy

\section{INTRODUCTION}

Oral pyogenic granuloma is a smooth or exophytic lesion on a sessile or pedunculated base covered with red hemorrhagic and compressible erythematous papules, which appear lobulated, warty, ulcerated, and covered by yellow fibrinous membrane (Neville et al., 2002; Regezi et al., 2003). Approximately $75 \%$ of oral pyogenic granuloma occurs in the gingiva (Brosens et al., 2015). It occurs in populations of all ages, but is frequently encountered in females in their 20s due to the increased levels of circulating hormones, such as estrogen and progesterone (Durairaj et al., 2011). Hence, the terms "pregnancy tumor" and "granuloma gravidarum" are widely employed (Neville et al., 2002). Multiple etiological factors lead to the formation of oral pyogenic granuloma. Chronic low-grade irritation (Kamal et al., 2012), hormonal factors, traumatic injury, and bacteria have been implicated as causative factors in the development of pyogenic granuloma (Janier, 1999; Kamal et al., 2012).

Excisional surgery remains the most common therapy for pyogenic granuloma(Esmeili et al., 2005). Recently, alternative conservative treatment protocols, such as cryosurgery (Ishida and Ramos-e-Silva, 1998; Wang and Goldberg, 2008), laser surgery, injection of absolute ethanol, and sodium tetradecyl sulfate sclerotherapy have been proposed (Ichimiya et al., 2004; Moon et al., 2005; Galeckas and Uebelhoer, 2009; Tsai et al., 2015). Other investigators also conducted a series of intralesional injections of corticosteroids for treating pyogenic granuloma, especially for highly recurrent lesions (Moon et al., 2005). Considering the etiological factors and clinical characteristics, we assume that initial periodontal therapy could eliminate irritants and yield high clinical efficacy. In this study, initial periodontal therapy was applied to manage the gingival pregnancy tumor and the clinical outcomes were evaluated.

\section{MATERIAL AND METHODS}

\section{Demographic data}

Thirty nine patients diagnosed with gingival pregnancy tumors between 2007 and 2015 were enrolled in this investigation. Tumors appeared during pregnancy and then expanded dramatically and still existed after child birth. Those who had epulis before pregnancy, serious systemic diseases, or took drugs containing fungal spores were excluded from this study. All patients reported poor oral hygiene and the oral lesions were described as soft or firm lumps with a purple, red, or pink surface. Among 39 cases, bleeding was observed in 29 , the base was pedunculated in 19 , and the surface was eroded or ulcerated in 21 . Four cases displaced adjacent teeth. The size of lesions ranged from 3.0 to $25.0 \mathrm{~mm}$ with a mean diameter of $12.3 \mathrm{~mm}$. 


\section{Initial periodontal treatment}

Initial periodontal therapy and promotion of dental health were conducted in 39 cases. Twenty five patients were repeatedly handled with periodontal maintenance treatment every 3 to 6 months. The remaining 14 cases demanded surgical treatment 1-3 months later. Before the operation, the tumors presented with no ulcerated surface and were significantly reduced in size. For those undergoing surgery, gingival lesions were excised approximately $1 \mathrm{~mm}$ to adjacent normal tissues and down to periosteum with curettage of the underlying bone if the periosteum was breached. The resulting surgical defect was covered with iodoform gauze and retained by sutures, which protected the wound from food and other debris and accelerated the process of healing. All patients received amoxicillin plus metronidazole or erythromycin plus metronidazole for those allergic to penicillin, and nonsteroidal anti-inflammatory agents. Patients were instructed to rinse frequently with hot salt water. The specimens were sent for histopathological confirmation.

\section{Statistical analysis}

Patients' age, lesion site, stage of pregnancy, diameter of lesions, and time of lesion remission were collected and analyzed descriptively. The statistical processes were executed using SPSS software (version 17.0, USA).

\section{RESULTS}

\section{Sites and onset time of gingival pregnancy tumor}

The lesions were located in the maxillary in 25 patients, mandible in 14, maxillary anterior in 26, maxillary posterior in 13, labio-buccal in 29, and lingual-palate in 13 . The age of the patients ranged from 22 to 38 years, with a mean age of 27.1 years. Approximately $94.9 \%$ of the lesions occurred in the second and third trimesters of pregnancy. Two cases developed gingival pregnancy tumors in early pregnancy, 24 during pregnancy metaphase, and 13 cases in late pregnancy. The gingival pregnancy tumors merely migrated into the gingival papilla.

\section{Diagnosis and treatment}

As shown in Figure 1A and B, extraoral examination was conducted and detected no deformity or lymph node enlargement. Intraoral examination revealed two large soft tissue masses on the left lingual (approximately $25 \mathrm{~mm}$ x $16 \mathrm{~mm}$ ) and labial $(4 \mathrm{~mm} \times 6$ $\mathrm{mm}$ ) side of the mandibular vestibule. The tumor growth was pedunculated and attached to the marginal gingiva interproximally between 42 and 43 , extending to lingual surfaces of 46 and 33. Radiograph of 42 and 43 revealed a slight amount of interdental alveolar crestal bone resorption, suggesting the effect of pressure (Figure 1C). During the first 3 months of treatment, the lesion was reduced significantly in response to initial periodontal therapy and the lesion of the labial side was eliminated completely. Supportive periodontal therapy was performed every 3 to 6 months and the size of lesions was minimized gradually, as illustrated in Figure 2. In certain cases, the lump was pedunculated and attached to the marginal gingiva interproximally between 11 and 21, extending to the buccal side (Figure 3A). Radiograph of 
11 and 21 revealed a slight amount of interdental alveolar crestal bone resorption, indicating the pressure effect (Figure 3B). The size of the lesion was minimized significantly with conservative surgery followed by initial periodontal therapy at 1 week, 1 month, and 6 months after surgery (Figure 4). Hematoxylin and eosin (H\&E) staining revealed ulcerated stratified squamous epithelium with fibrovascular stroma. The stroma consisted of a substantial amount of budding capillaries, plump fibroblasts, areas of extravasated blood and chronic inflammatory cell infiltration (Figure 5).


Figure 1. Appearance of the labial (A) and lingual (B) tumors. Radiograph of the tumor indicating pressure effect (C).
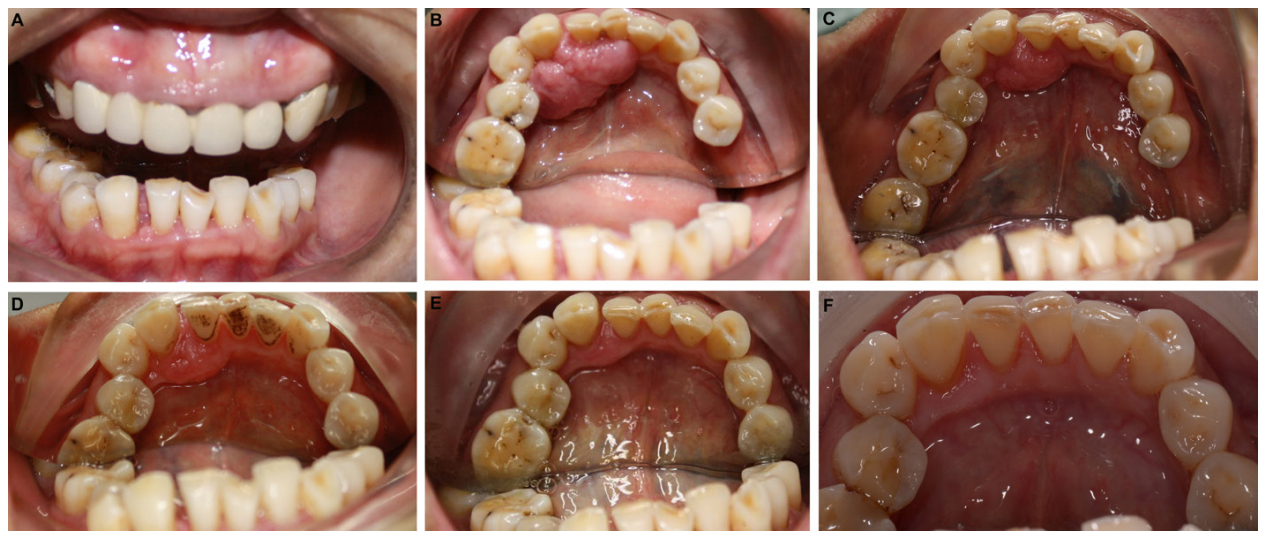

Figure 2. Appearance of the gingival tumors after 3 months (A and B), 9 months (C), 16 months (D), 20 months $(\mathbf{E})$, or 6 years $(\mathbf{F})$ of therapy.


Figure 3. Appearance of a lump involving anterior maxilla (A). Radiograph of the tumor showing a slight amount of alveolar crestal bone resorption (B). 

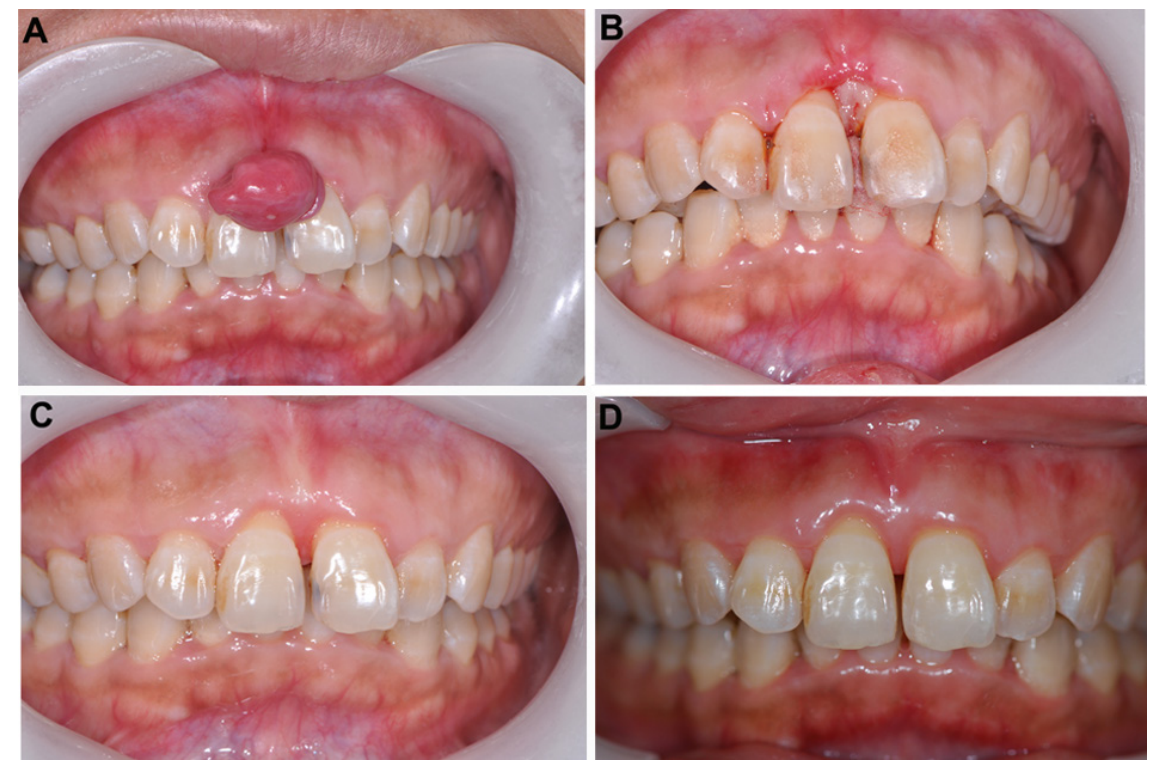

Figure 4. Two months after initial periodontal therapy, surgical treatment was executed (A). Appearance of the tumors after 1 week (B), 1 month (C), and 6 months (D) of therapy.



Figure 5. H\&E staining showing dense chronic inflammatory cell infiltration (100X).

\section{Clinical efficacy of initial periodontal treatment}

Following initial periodontal therapy, gingival pregnancy tumors were gradually eliminated in 25 cases. During early stages of treatment, the tumors were reduced quickly and then at a slower pace with more dense texture over the course of treatment (Table 1). In 
detail, three cases had a tumor size $<5 \mathrm{~mm}$ and the tumors were reduced in size in a mean time of 3.6 months. Four patients had a tumor size ranging from 5 to $10 \mathrm{~mm}$ and the tumors were eliminated in an average time of 7.5 months. Eleven cases had a tumor size of 10-15 mm were reduced in a mean time of 10.2 months. Six patients with a tumor size $15-20 \mathrm{~mm}$ were treated in a mean time of 15 months. Only one case with a tumor size $>20 \mathrm{~mm}$ required 20 months of treatment. Fourteen cases were subject to initial periodontal therapy and then underwent surgical biopsy after 1 to 3 months. The biopsy outcomes confirmed the diagnosis of pyogenic granuloma. During subsequent follow-up (range: $0.5-8$ years), no recurrence was reported in all patients.

Table 1. Diameter of lesions and treatment effect.

\begin{tabular}{l|c|c|c|c}
\hline Diameter & Number of cases & Surgery group & Initial periodontal therapy group & Mean time of lesion removal (months) \\
\hline$<5 \mathrm{~mm}$ & 3 & 0 & 3 & $3.6 \pm 0.57$ \\
\hline $5-10 \mathrm{~mm}$ & 7 & 3 & 4 & $7.5 \pm 1.3$ \\
\hline $10-15 \mathrm{~mm}$ & 18 & 7 & 11 & $10.2 \pm 0.98$ \\
\hline $15-20 \mathrm{~mm}$ & 10 & 4 & 6 & $15 \pm 2.4$ \\
\hline$>20 \mathrm{~mm}$ & 1 & 0 & 1 & 20 \\
\hline
\end{tabular}

\section{DISCUSSION}

Pregnancy tumor is a typical type of pyogenic granuloma, which cannot be clinically and histologically distinguished from pyogenic granuloma occurring in non-pregnant women (Mealey and Moritz, 2003). Daley et al. (1991) indicated that clinical diagnosis of pregnancy tumor is valid by describing a pyogenic granuloma occurring in pregnancy because the distinct lesion is described based on the etiology, biological behavior, and treatment protocol rather than on the histological features. In this study, the lesions of all cases were observed during pregnancy and accompanied by poor oral hygiene. Obvious calculus was noted surrounding the tumors. Among 39 cases, 14 were diagnosed with pyogenic granuloma by pathological biopsy and the other 25 were handled with initial periodontal therapy and dental health promotion, which equally decreased the tumor size.

In terms of the distribution of gingival pregnancy tumors, the maxilla, anterior gingiva, and labio-buccal gingiva were more frequently involved compared with the mandible, posterior gingiva, and lingual-palatal gingiva, which is consistent with previous findings (Chamani et al., 2009; Krishnapillai et al., 2012). In this study, the mean age of the enrolled patients was 27.1 years, similar to that of the study by Cardoso et al. (2013). A majority of the oral tumors occurred in the second and third trimesters of pregnancy, in accordance with the findings of another previous study (Terezhalmy et al., 2001).

The pathogenesis of gingival pregnancy tumor occurs due to the combination of periodontal pathogens, local irritants, and multiple female hormones. Firstly, calculus-induced gingival irritation might be the predisposing factor for the development of gingival pyogenic granuloma. The microulceration from these irritants in an inflammatory gingiva allows for the ingress of low virulent oral microflora into the gingival connective tissues, which evokes an exaggerated vascular hyperplastic response in the connective tissues resulting in the formation of pyogenic granuloma (Lawoyin et al., 1997). Secondly, progesterone, estrogen, and human chorionic gonadotropin are three major hormones secreted by the placenta (Simpson and MacDonald, 1981). Human chorionic gonadotropin is produced in large amounts during 
the first 10 weeks of pregnancy, after which the levels fall dramatically. However, during pregnancy the concentrations of progesterone and estrogen rise steadily until childbirth (Mariotti, 1994). Sex hormones manifest a variety of biological and immunological effects. Estrogen accelerates wound healing by stimulating the production of nerve growth factor in macrophages, granulocyte-colony stimulating factors in keratinocytes, and basic fibroblast growth factors and transforming growth factor $\beta 1$ in fibroblasts, leading to the formation of granulated tissues. In addition, estrogen is able to enhance the generation of vascular endothelial growth factor (VEGF) in macrophages, which is antagonized by androgens and probably correlates with the development of pyogenic granuloma during pregnancy (Kanda and Watanabe, 2005). Progesterone functions as an immunosuppressant in the gingival tissue of pregnant women, preventing a rapid acute inflammatory reaction against plaque, but allowing for increased chronic tissue reaction and resulting in exaggerated inflammation (Durairaj et al., 2011). Lastly, during pregnancy, female sex hormones induce clinico-microbiological changes, particularly an increased number of the periodontal pathogens Porphyromonas gingivalis and Prevotella intermedia, that affect the target cells, including keratinocytes and fibroblasts, in the gingiva (Carrillo-de-Albornoz et al., 2010). If this condition is left untreated and is accompanied by poor oral hygiene, the exaggerated tissue responses further progress into pregnancy tumor. The tissue response is attributed to the stimulation of fibroblasts to produce fibroblast growth factor, transforming growth factor, and VEGF. High angiogenic activity demonstrated by immunohistochemistry implied the clinical presentation of bleeding tendency (Whitaker et al., 1994). Female sex hormones including progesterone and estrogen are neither necessary nor sufficient to produce gingival changes by themselves. However, they may alter periodontal tissue responses to microbial plaque and thus indirectly contribute to periodontal disease.

Conservative surgical excision is recommended for small, painless, non-bleeding lesions, such as plaque, calculus, and foreign materials. Excision of the gingival lesions is performed approximately $1 \mathrm{~mm}$ to the normal tissues and above the periosteum through scaling and root planning of adjacent teeth to remove all visible sources of irritation (Esmeili et al., 2005). However, the surgical approach can lead to tissue defects and severe gingival injury, especially when the pregnancy tumor is located in the esthetic area (Joda, 2012). In this study, after initial periodontal therapy and oral health maintenance, 14 tumors were decreased considerably in patients undergoing surgery. The surgical wound was smaller and intraoperative and postoperative bleeding volume was diminished. Displacement of the adjacent teeth was seen in four cases possibly due to lesion expansion. However, the adverse event was recovered gradually as the volume of lesions was minimized by initial periodontal treatment or surgery. Two patients recovered completely following initial periodontal treatment and another 2 were partially improved. Initial periodontal therapy yields mild oral trauma, which is especially suitable for patients who are reluctant or afraid of undergoing surgery. More importantly, it is an appropriate choice for those with a health condition too poor to receive surgery, such as lactating women. However, the treatment time is relatively longer compared with alternative non-surgical or conservative options. According to the findings of this clinical trial, the authors are willing to share the following experience. First, to achieve high clinical efficacy, topical irritant factors should be thoroughly eliminated throughout the clinical procedures. Second, the patients are required to pay a return visit to receive maintenance therapy to consolidate the clinical effect and identify new problems. Last, the patients are advised to pay close attention to oral hygiene care and instructed to correctly wash their teeth by using various tools, such as 
dental floss, dental water jet, toothbrush, and toothpaste.

Taken together, although both surgical and conservative therapies have been applied to treat gingival pregnancy tumor, the risk of recurrence is still high (Reddy et al., 2014). Gingival pregnancy tumor arises in response to various stimuli, such as low-grade local irritation, periodontal pathogens, and a variety of sex hormones, so removal of causative irritants serves as the major line of treatment. In this study, no recurrence was observed over a follow-up period of 0.5-8 years, which indicates the high clinical efficacy and safety of initial periodontal therapy. Considering the complex causes of gingival pregnancy tumor, maintenance of oral hygiene, removal of dental plaques, and use of soft toothbrushes are the routine preventive measures during the antenatal period (Sills et al., 1996).

\section{Conflicts of interest}

The authors declare no conflict of interest.

\section{REFERENCES}

Brosens LA, Hackeng WM, Offerhaus GJ, Hruban RH, et al. (2015). Pancreatic adenocarcinoma pathology: changing "landscape". J. Gastrointest. Oncol. 6: 358-374.

Cardoso JA, Spanemberg JC, Cherubini K, Figueiredo MA, et al. (2013). Oral granuloma gravidarum: a retrospective study of 41 cases in Southern Brazil. J. Appl. Oral Sci. 21: 215-218.

Carrillo-de-Albornoz A, Figuero E, Herrera D and Bascones-Martínez A (2010). Gingival changes during pregnancy: II. Influence of hormonal variations on the subgingival biofilm. J. Clin. Periodontol. 37: 230-240. http://dx.doi. org/10.1111/j.1600-051X.2009.01514.x

Chamani G, Navvabi N and Abdollahzadeh SH (2009). The frequency of pregnancy tumor in pregnant mothers. Dent. J. Shiraz Med. Sci. Univ. 10: 79-82.

Daley TD, Nartey NO and Wysocki GP (1991). Pregnancy tumor: an analysis. Oral Surg. Oral Med. Oral Pathol. 72: 196-199. http://dx.doi.org/10.1016/0030-4220(91)90163-7

Durairaj J, Balasubramanian K, Rani PR, Sagili H, et al. (2011). Giant lingual granuloma gravidarum. J. Obstet. Gynaecol. 31: 769-770. http://dx.doi.org/10.3109/01443615.2011.615419

Esmeili T, Lozada-Nur F and Epstein J (2005). Common benign oral soft tissue masses. Dent. Clin. North Am. 49: 223240, x. http://dx.doi.org/10.1016/j.cden.2004.07.001

Galeckas KJ and Uebelhoer NS (2009). Successful treatment of pyogenic granuloma using a 1,064-nm laser followed by glycerin sclerotherapy. Dermatol. Surg. 35: 530-534. http://dx.doi.org/10.1111/j.1524-4725.2009.01081.x

Ichimiya M, Yoshikawa Y, Hamamoto Y and Muto M (2004). Successful treatment of pyogenic granuloma with injection of absolute ethanol. J. Dermatol. 31: 342-344. http://dx.doi.org/10.1111/j.1346-8138.2004.tb00682.x

Ishida CE and Ramos-e-Silva M (1998). Cryosurgery in oral lesions. Int. J. Dermatol. 37: 283-285. http://dx.doi. org/10.1046/j.1365-4362.1998.00426.x

Janier M (1999). [Infection and angiomatous cutaneous lesions]. J. Mal. Vasc. 24: 135-138.

Joda T (2012). Esthetic management of mucogingival defects after total excision in a case of pyogenic granuloma. Eur. J. Esthet. Dent. 7: 110-119.

Kamal R, Dahiya P and Puri A (2012). Oral pyogenic granuloma: Various concepts of etiopathogenesis. J. Oral Maxillofac. Pathol. 16: 79-82. http://dx.doi.org/10.4103/0973-029X.92978

Kanda N and Watanabe S (2005). Regulatory roles of sex hormones in cutaneous biology and immunology. J. Dermatol. Sci. 38: 1-7. http://dx.doi.org/10.1016/j.jdermsci.2004.10.011

Krishnapillai R, Punnoose K, Angadi PV and Koneru A (2012). Oral pyogenic granuloma--a review of 215 cases in a South Indian Teaching Hospital, Karnataka, over a period of 20 years. Oral Maxillofac. Surg. 16: 305-309. http:// dx.doi.org/10.1007/s10006-012-0315-z

Lawoyin JO, Arotiba JT and Dosumu OO (1997). Oral pyogenic granuloma: a review of 38 cases from Ibadan, Nigeria. Br. J. Oral Maxillofac. Surg. 35: 185-189. http://dx.doi.org/10.1016/S0266-4356(97)90561-1

Mariotti A (1994). Sex steroid hormones and cell dynamics in the periodontium. Crit. Rev. Oral Biol. Med. 5: 27-53.

Mealey BL and Moritz AJ (2003). Hormonal influences: effects of diabetes mellitus and endogenous female sex steroid 
hormones on the periodontium. Periodontol. 2000 32: 59-81. http://dx.doi.org/10.1046/j.0906-6713.2002.03206.x

Moon SE, Hwang EJ and Cho KH (2005). Treatment of pyogenic granuloma by sodium tetradecyl sulfate sclerotherapy. Arch. Dermatol. 141: 644-646. http://dx.doi.org/10.1001/archderm.141.5.644

Neville B, Damm D and Allen C (2002). Oral and maxillofacial surgery. 2 ed. Published by Saunders, Philadelphia, USA.

Reddy NR, Kumar PM, Selvi T and Nalini HE (2014). Management of recurrent post-partum pregnancy tumor with localized chronic periodontitis. Int. J. Prev. Med. 5: 643-647.

Regezi J, Sciubba J and Jordan R (2003). Oral pathology: clinical pathological considerations, 4 ed. Published by Saunders, Philadelphia, USA.

Sills ES, Zegarelli DJ, Hoschander MM and Strider WE (1996). Clinical diagnosis and management of hormonally responsive oral pregnancy tumor (pyogenic granuloma). J. Reprod. Med. 41: 467-470.

Simpson ER and MacDonald PC (1981). Endocrine physiology of the placenta. Annu. Rev. Physiol. 43: 163-188. http:// dx.doi.org/10.1146/annurev.ph.43.030181.001115

Terezhalmy GT, Riley CK and Moore WS (2001). Focal epithelial hyperplasia (Heck's disease). Quintessence Int. 32: 664-665.

Tsai KY, Wang WH, Chang GH and Tsai YH (2015). Treatment of pregnancy-associated oral pyogenic granuloma with life-threatening haemorrhage by transarterial embolisation. J. Laryngol. Otol. 129: 607-610. http://dx.doi. org $/ 10.1017 / \mathrm{S} 0022215115001176$

Wang SQ and Goldberg LH (2008). Treatment of recurrent pyogenic granuloma with excision and frozen section for margin control. Dermatol. Surg. 34: 1115-1116.

Whitaker SB, Bouquot JE, Alimario AE and Whitaker TJ, Jr. (1994). Identification and semiquantification of estrogen and progesterone receptors in pyogenic granulomas of pregnancy. Oral Surg. Oral Med. Oral Pathol. 78: 755-760. http:// dx.doi.org/10.1016/0030-4220(94)90092-2 